\title{
Micafungin versus caspofungin in the treatment of Candida glabrata infection: a case report
}

\author{
Shoko Merrit Yamada ${ }^{1 *}$, Yusuke Tomita ${ }^{1}$, Tomotsugu Yamaguchi ${ }^{2}$ and Toshiaki Matsuki ${ }^{2}$
}

\begin{abstract}
Background: Micafungin and caspofungin, which are both echinocandins, elicit their antifungal effects by suppressing the synthesis of $\beta$-D-glucan, an essential component of fungal cell walls. If micafungin is not effective against a fungal infection, is it unreasonable to switch to caspofungin?

Case presentation: An 80-year-old Asian man presented to our hospital with brain and lung abscesses. Klebsiella pneumonia and Escherichia coli were identified by sputa culture and Streptococcus mitis was identified in the brain abscess culture obtained by drainage surgery. He was treated with antibiotics and both abscesses shrank after the treatment. But he continued to have a high fever and Candida glabrata was identified by blood culture. The origin of the infection was not clarified and micafungin was administered intravenously. The fungus showed poor susceptibility to micafungin; we then switched the antifungal from micafungin to caspofungin. After caspofungin treatment, his body temperature remained below $37^{\circ} \mathrm{C}$ and his $\beta$-D-glucan levels decreased remarkably.

Conclusions: In vitro, micafungin is considered more effective against C. glabrata because its minimum inhibitory concentration against C. glabrata is lower than that of caspofungin. However, in vivo, there is no significantly different effect between the two drugs. When micafungin is not effective against candidiasis, a switch to caspofungin might be applicable because the pharmacokinetics in each echinocandin is slightly different.
\end{abstract}

Keywords: Candidiasis, Caspofungin, Echinocandins, Fungal infection, Micafungin, Case report

\section{Background}

Antifungal agents that are currently used for deep fungal infections include polyene macrolide derivatives (amphotericin B), azole antifungals (imidazoles and triazoles), and echinocandins. Echinocandins are recommended as the primary therapy for invasive candidiasis [1], and resistance to echinocandins remains low, at less than $3 \%$, in Candida albicans [2]. However, cases of echinocandin-resistant Candida glabrata are increasing with cross-resistance to azole antifungal agents [3, 4]. Here, we report a case of successful treatment for C. glabrata infection that showed poor sensitivity to micafungin (MCFG), which is an echinocandin.

\footnotetext{
* Correspondence: smyamada@med.teikyo-u.ac.jp

${ }^{1}$ Department of Neurosurgery, Teikyo University Mizonokuchi Hospital, 3-8-3

Mizonokuchi, Takatsu-ku, Kawasaki, Kanagawa 213-8507, Japan

Full list of author information is available at the end of the article

\section{Case presentation}

An 80-year-old Asian man presented to our hospital with left hemiparesis; a manual muscle test revealed motor weaknesses of $2+/ 5$ in his upper limbs and $4 / 5$ in his lower limbs. Magnetic resonance imaging (MRI) demonstrated a brain abscess in his right frontal lobe (motor area), and both a chest X-ray and a computed tomography (CT) scan showed a lung abscess in the lower lobe of his right lung (Fig. 1a, day 0). He had undergone partial resection of his stomach due to gastric cancer 11 years ago, but he did not take anti-cancer drugs and no recurrence was identified. The drugs he had been taking were $50 \mathrm{mg}$ of sodium ferrous citrate, $1500 \mu \mathrm{g}$ vitamin B12, $20 \mathrm{mg}$ esomeprazole, and $2.5 \mathrm{mg}$ amlodipine. His medical history did not indicate an immune deficiency disorder. No organ dysfunction was indicated from laboratory data. But mild anemia was identified: his hemoglobin value was $11.9 \mathrm{~g} / \mathrm{dl}$ (normal range 13.5 to 17.6 ), hematocrit $35.0 \%$ (39.8 to 51.8 ), 


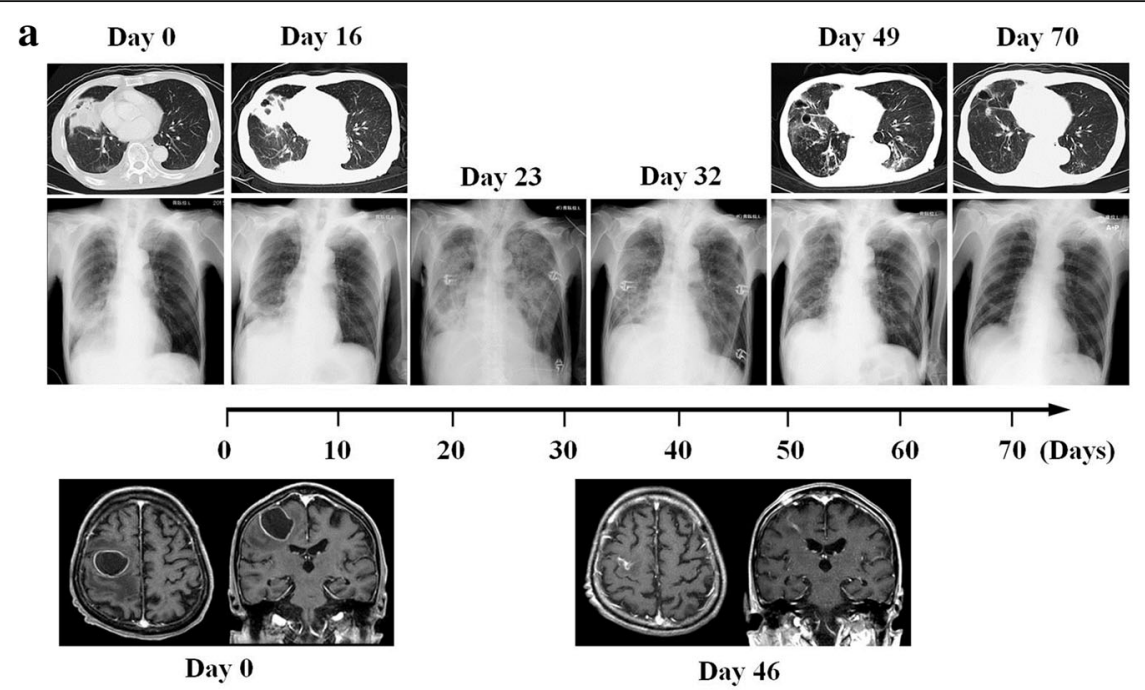

b

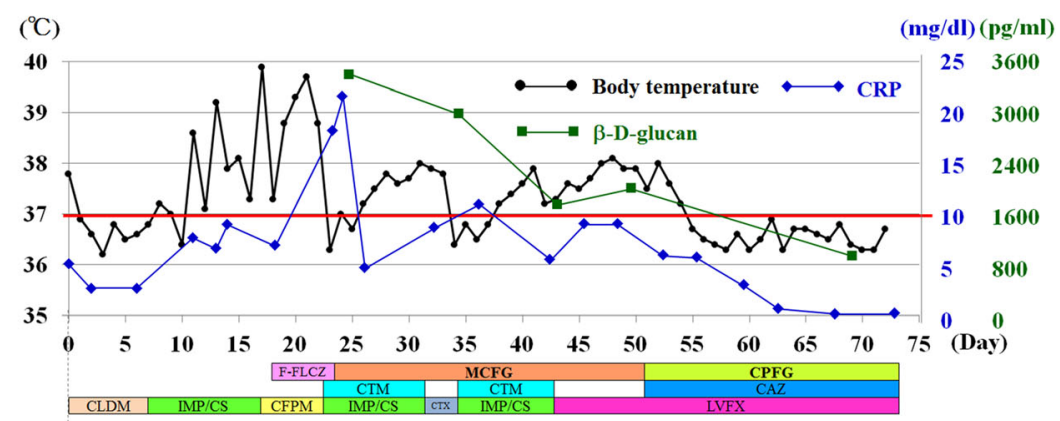

Fig. 1 Chronological changes in the brain and lung abscesses. a Image studies: top, chest computed tomography scan; middle, chest X-ray; and bottom, head magnetic resonance imaging with contrast medium. b Alterations in body temperature (black), C-reactive protein levels (blue), and $\beta$-D-glucan levels (green) in response to the changes in antibiotics and antifungals. Daily doses: clindamycin $600 \mathrm{mg}$, twice a day; imipenem/cilastatin sodium $0.5 \mathrm{~g}$, twice a day; cefepime dihydrochloride hydrate $1 \mathrm{~g}$, twice a day; cefotiam hydrochloride $1 \mathrm{~g}$, twice a day; levofloxacin hydrate 500 mg, once a day; ceftazidime hydrate $1 \mathrm{~g}$, twice a day; fosfluconazole $800 \mathrm{mg}$, once a day on the first day, and $400 \mathrm{mg}$, once a day after second day; micafungin sodium $150 \mathrm{mg}$, once a day; and caspofungin acetate $70 \mathrm{mg}$, once a day on the first day, and $50 \mathrm{mg}$, once a day after second day. The red horizontal line in the graph indicates the body temperature level of $37^{\circ} \mathrm{C}$ and CRP level of $10 \mathrm{mg} / \mathrm{dl}$. CAZ ceftazidime hydrate, CFPM cefepime dihydrochloride hydrate, CLDM clindamycin, CPFG caspofungin acetate, CRP C-reactive protein, CTM cefotiam hydrochloride, CTX cefotaxime, F-FLCZ fosfluconazole, IMP/CS imipenem/Cilastatin sodium, LVFX levofloxacin hydrate, MCFG micafungin sodium

iron $(\mathrm{Fe}) 49 \mathrm{mg} / \mathrm{dl}$ (50 to 170), and ferritin $98.9 \mathrm{ng} / \mathrm{ml}$ (21 to 282). Because he was not able to eat enough food for 1 week before admission, his nutritional status was poor: serum total protein $6.1 \mathrm{~g} / \mathrm{dl}$ (normal range 6.7 to 8.3), albumin $3.2 \mathrm{~g} / \mathrm{dl}$ (3.8 to 5.1), total cholesterol 148 $\mathrm{mg} / \mathrm{dl}$ (150 to 219 ), and triglyceride $126 \mathrm{mg} / \mathrm{dl}$ (50 to $150)$.

\section{Days 0-10}

On admission, he was administered clindamycin (CLDM; $600 \mathrm{mg}$ twice a day) because we were considering aspiration pneumonia. However, this was switched to imipenem/cilastatin sodium (IMP/CS; $0.5 \mathrm{~g}$ twice a day) on day 6 post-admission because Klebsiella pneumoniae and Escherichia coli were identified by sputa culture (Fig. 1b).

\section{Days 11-20}

After the antibiotic was changed to IMP/CS, his body temperature gradually elevated, his serum C-reactive protein (CRP) level also increased to $7.8 \mathrm{mg} / \mathrm{dl}$ (normal range $<0.3 \mathrm{mg} / \mathrm{dl}$ ), and his left hemiparesis worsened. To address these symptoms, a surgical aspiration of his brain abscess was performed under local anesthesia on day 12. Although his left hemiparesis mildly improved after the surgery and the follow-up chest X-ray and CT scan showed that he was recovering from pneumonia (Fig. 1a, Day 16), high fever continued after the surgery in spite of the IMP/CS treatment. Streptococcus mitis was identified in the brain abscess culture, and a blood culture performed on day 16 indicated he had a simultaneous fungal infection. The antibiotic was changed to cefepime dihydrochloride hydrate (CFPM; 1 g twice a 
day) targeting a Gram-positive coccus and fosfluconazole (F-FLCZ; $800 \mathrm{mg}$ once a day on the first day and $400 \mathrm{mg}$ once a day after second day) treatment was added for fungal infection. Despite these treatments, he still had a high body temperature $\left(39.7{ }^{\circ} \mathrm{C}\right)$ on day 20 (Fig. 1b). At this time, a central venous catheter was inserted in his right subclavian vein for parenteral nutrition because he was unable to get sufficient nutrients through oral intake.

\section{Days 21-30}

On day 21, 4 days after CFPM treatment started, his body temperature elevated again to $39.6{ }^{\circ} \mathrm{C}$ and his serum CRP level drastically increased. He had difficulty in breathing and was intubated with artificial respirator assistance because his chest X-ray showed diffuse interstitial pneumonia (Fig. 1a, Day 23) and E. coli was still identified in sputa culture. The antibiotic was switched back to IMP/CS (0.5 g twice a day) targeting E.coli, and cefotiam hydrochloride (CTM; $1 \mathrm{~g}$ twice a day) was used for brain abscess treatment because we considered it had good transitivity of the antibiotic to the cerebrospinal fluid. In addition, C. glabrata, which is resistant to F-FLCZ, was detected via the blood culture performed on day 16 , and his level of $\beta$-D-glucan was extremely high at $3634 \mathrm{pg} / \mathrm{ml}$ (normal range $<20 \mathrm{pg} / \mathrm{ml}$ ). Then, he received combination treatments of MCFG (150 mg once a day), IMP/CS (0.5 g twice a day), and CTM (1 g twice a day) because of multiple infections resulting from fungi and from both Gram-negative bacillus and Gram-positive coccus bacteria (Fig. 1b).

\section{Days 31-50}

A chest X-ray on day 32 displayed an improvement in his diffuse interstitial pneumonia (Fig. 1a), and he was extubated. His antibiotic treatments with IMP/CS and CTM were changed to cefotaxime (CTX; $1 \mathrm{~g}$ twice a day) because two strong antibiotics were used for 1 week. However, his CRP rose rapidly to $7.4 \mathrm{mg} / \mathrm{dl}$ from 4.7, and the antibiotic was switched back to IMP/CS $(0.5$ $\mathrm{g}$ twice a day) and CTM (1 g twice a day). Another 1 week of combination treatment of IMP/CS and CTM was performed. His body temperature was constantly elevated over $37.0{ }^{\circ} \mathrm{C}$ and his CRP never decreased less than $5.0 \mathrm{mg} / \mathrm{dl}$. Then, levofloxacin hydrate (LVFX; 500 mg once a day), which was classified as quinolone antibiotic, was applied from day 43. During the period of MCFG administration, his body temperature fluctuated between 37 and $38{ }^{\circ} \mathrm{C}$ and his CRP level varied between 5 and $10 \mathrm{mg} / \mathrm{dl}$. On day 43, his level of $\beta$-D-glucan decreased to $1732 \mathrm{pg} / \mathrm{ml}$. MRI on day 46 revealed that his brain abscess had almost completely disappeared (Fig. 1a, day 46) and a chest X-ray demonstrated amelioration of his pneumonia (Fig. 1a, Day 49). However, on day 49, his body temperature climbed to $38{ }^{\circ} \mathrm{C}$, his CRP level increased to $9.8 \mathrm{mg} / \mathrm{dl}$, and his $\beta$-D-glucan level elevated to $1947 \mathrm{pg} / \mathrm{ml}$. To address these symptoms, on day 51, the MCFG was switched to caspofungin (CPFG; $70 \mathrm{mg}$ once a day on the first day and $50 \mathrm{mg}$ once a day after second day) and ceftazidime hydrate (CAZ; 1 g twice a day) treatment was added (Fig. 1b).

\section{Days 51-72}

Three days after initiating the CPFG and CAZ treatments, his body temperature began decreasing and never exceeded $37{ }^{\circ} \mathrm{C}$ after day 55. His CRP level also rapidly decreased after the initiation of CPFG treatment; it dropped within the normal range and did not increase again after day 63. The central venous catheter was removed on day 65 because he could ingest fully. His $\beta$-D-glucan level decreased to $1000 \mathrm{pg} /$ $\mathrm{ml}$ (Fig. 1b), and C. glabrata was not identified in the blood culture performed on day 69 .

At day 70, a chest X-ray and CT scan demonstrated successful treatment of his lung abscess and pneumonia (Fig. 1a). When he was discharged from our hospital on day 73 , he had regained his motor function (4-/5 in his left upper limb and 5/5 in his left lower limb by a manual muscle test).

\section{Conclusions}

In our patient, the risk factors for candidemia included partial resection of stomach causing Fe deficiency anemia, malnutritional status on admission, age, and the long-term usage of antibiotics. C. glabrata is one of the prevalent causes of candidemia, and it exhibits poor susceptibility to azole antifungals, acquiring resistance rapidly $[5,6]$. Instead, echinocandins are recommended when Candida infection is suspected [1]. Anidulafungin (ANFG), CPFG, and MCFG are all echinocandins with similar antifungal activity spectra [7-9], but only CPFG and MCFG are authorized in Japan. In in vitro studies, the minimum inhibitory concentration against C. glabrata was higher in CPFG than in ANFG or MCFG, and MCFG was the most potent agent against C. glabrata [9-11]. However, the clinical outcomes of patients with systemic candidiasis due to C. glabrata were not significantly different if they were treated with MCFG or with CPFG [12].

The level of serum $\beta$-D-glucan increased in spite of MCFG treatment in our patient; however, we switched the antifungal to CPFG, another echinocandin, rather than changing to a different class of antifungals because echinocandins suppress $\beta$-D-glucan synthesis better than any other antifungals [13]. Notably, CPFG treatment was dramatically effective in our case. Spreghini et al. found that, in the absence of human serum, the susceptibilities of C. glabrata to MCFG and to CPFG 
were not statistically different from one another, whereas, in the presence of human serum, C. glabrata was more susceptible to CPFG than to MCFG $(p<0.05)$ [14]. In their study using mice, CPFG started to be effective after 2 days of treatment, while after 6 days, the lowest effective doses were $0.25,1$, and $5 \mathrm{mg} / \mathrm{kg} /$ day for CPFG, MCFG, and ANFG, respectively [14]. Our case clearly demonstrates the effectiveness of CPFG in a patient with C. glabrata infection that showed resistance to MCFG. In echinocandin pharmacokinetics, the protein-binding form of the drug constitutes $99.8 \%$ in MCFG and $97 \%$ in CPFG, and the clearance of CPFG from the human body is much slower than that of MCFG [13]. The lower percentage of protein-binding form and slower excretion of CPFG may help it maintain an effective drug concentration against C. glabrata for longer than MCFG.

In fact, invasive candidiasis was successfully treated by CPFG in our case, but we do not conclude that CPFG is more effective than MCFG for C. glabrata infection because the in vivo antifungal activity, pharmacokinetics, and toxicity profiles are slightly different in each echinocandin [15]. We considered that the sensitivity pattern of the Candida to echinocandins in our patient was that the C. glabrata in our patient was basically sensitive to echinocandins, and it was more sensitive to CPFG than to MCFG. Some advantages of MCFG treatment are that serious side effects rarely occur and it can be used when the patient has mild to severe hepatic dysfunction [13, 16]. Unfortunately, CPFG is the only echinocandin for which dosage reduction is recommended if patients have even mild hepatic dysfunction [1]. Therefore, MCFG should still be the first drug choice for candidemia; however, if it is not effective, early replacement with CPFG should be considered, rather than adding another kind of antifungal agent.

\section{Abbreviations}

ANFG: Anidulafungin; CAZ: Ceftazidime hydrate; CFPM: Cefepime dihydrochloride hydrate; CLDM: Clindamycin; CPFG: Caspofungin; CRP: Serum C-reactive protein; CT: Computed tomography; CTM: Cefotiam hydrochloride; CTX: Cefotaxime; Fe: Iron; F-FLCZ: Fosfluconazole; IMP/CS: Imipenem/cilastatin sodium;

LVFX: Levofloxacin hydrate; MCFG: Micafungin; MRI: Magnetic resonance imaging

\section{Acknowledgements}

The authors express our gratitude to the Edanz Group for copyediting our manuscript.

\section{Funding}

This case report did not receive any funding support.

\section{Availability of data and materials}

The data and reproducible materials described in this manuscript are freely available to any scientist wishing to use them.

\section{Authors' contributions}

SMY took care of the patient and wrote the manuscript. YT took care of the patient and analyzed the data of the patient. TY and TM contributed to collect the information on anti-fungal drugs. All authors read and approved the final manuscript.

\section{Competing interests}

The authors declare that they have no competing interests.

\section{Consent for publication}

Written informed consent was obtained from the patient for publication of this case report and any accompanying images. A copy of the written consent is available for review by the Editor-in-Chief of this journal.

\section{Author details}

'Department of Neurosurgery, Teikyo University Mizonokuchi Hospital, 3-8-3 Mizonokuchi, Takatsu-ku, Kawasaki, Kanagawa 213-8507, Japan. ${ }^{2}$ Department of Pharmacy, Teikyo University Mizonokuchi Hospital, 3-8-3 Mizonokuchi, Takatsu-ku, Kawasaki, Kanagawa 213-8507, Japan.

Received: 2 July 2016 Accepted: 11 October 2016

Published online: 08 November 2016

\section{References}

1. Pappas PG, Kauffman CA, Andes D, Benjamin Jr DK, Calandra TF, Edwards Jr JE, Filler SG, Fisher JF, Kullberg BJ, Ostrosky-Zeichner L, Reboli AC, Rex JH, Walsh TJ, Sobel JD. Clinical practice guidelines for the management of candidiasis: 2009 update by the Infectious Diseases Society of America. Infectious Diseases Society of America. Clin Infect Dis. 2009;48:503-35.

2. Castanheira M, Woosley LN, Diekema DJ, Messer SA, Jones RN, Pfaller MA. Low prevalence of $f k s 1$ hot spot 1 mutations in a worldwide collection of Candida strains. Antimicrob Agents Chemother. 2010;54:2655-9.

3. Alexander $B D$, Johnson MD, Pfeiffer CD, Jiménez-Ortigosa C, Catania J, Booker R, Castanheira M, Messer SA, Perlin DS, Pfaller MA. Increasing echinocandin resistance in Candida glabrata: clinical failure correlates with presence of FKS mutations and elevated minimum inhibitory concentrations. Clin Infect Dis. 2013;56:1724-32

4. Pham CD, lqbal N, Bolden CB, Kuykendall RJ, Harrison LH, Farley MM, Schaffner W, Beldavs ZG, Chiller TM, Park BJ, Cleveland AA, Lockhart SR. Role of FKS Mutations in Candida glabrata: MIC values, echinocandin resistance, and multidrug resistance. Antimicrob Agents Chemother. 2014;58:4690-6.

5. Pfaller MA, Moet GJ, Messer SA, Jones RN, Castanheira M. Geographic variations in species distribution and echinocandin and azole antifungal resistance rates among Candida bloodstream infection isolates: report from the SENTRY Antimicrobial Surveillance Program (2008 to 2009). J Clin Microbiol. 2011:49:396-9.

6. Quindós G. Epidemiology of candidaemia and invasive candidiasis. A changing face. Rev Iberoam Micol. 2014;31:42-8.

7. Cappelletty D, Eiselstein-McKitrick K. The echinocandins. Pharmacotherapy. 2007:27:369-88.

8. Pound MW, Townsend ML, Drew RH. Echinocandin pharmacodynamics: review and clinical implications. J Antimicrob Chemother. 2010;65:1108-18.

9. Smith RP, Baltch A, Bopp LH, Ritz WJ, Michelsen PP. Post-antifungal effects and time-kill studies of anidulafungin, caspofungin, and micafungin against Candida glabrata and Candida parapsilosis. Diagn Microbiol Infect Dis. 2011; 71:131-8.

10. Clancy CJ, Huang $\mathrm{H}$, Cheng S, Derendorf $\mathrm{H}$, Nguyen $\mathrm{MH}$. Characterizing the effects of caspofungin on Candida albicans, Candida parapsilosis, and Candida glabrata isolates by simultaneous time-kill and postantifungal-effect experiments. Antimicrob Agents Chemother. 2006;50:2569-72.

11. Gil-Alonso S, Jauregizar N, Cantón E, Eraso E, Quindós G. In vitro fungicidal activities of anidulafungin, caspofungin, and micafungin against Candida glabrata, Candida bracarensis, and Candida nivariensis evaluated by time-kill studies. Antimicrob Agents Chemother. 2015;59:3615-8.

12. Pappas PG, Rotstein CM, Betts RF, Nucci M, Talwar D, De Waele JJ, Vazquez JA, Dupont BF, Horn DL, Ostrosky-Zeichner L, Reboli AC, Suh B, Digumarti R, Wu C, Kovanda LL, Arnold LJ, Buell DN. Micafungin versus caspofungin for treatment of candidemia and other forms of invasive candidiasis. Clin Infect Dis. 2007;45:883-93.

13. Kohno S, Kakeya H, Miyazaki Y. A new class of antifungal agent Candins. Nihon Kagakuryoho Gakkai Zassi. 2002;50:839-47 [in Japanese].

14. Spreghini E, Orlando F, Sanguinetti M, Posteraro B, Giannini D, Manso E, Barchiesi F. Comparative Effects of Micafungin, Caspofungin, and Anidulafungin 
against a Difficult-To-Treat Fungal Opportunistic Pathogen, Candida glabrata. Antimicrob Agents Chemother. 2012;56:1215-22.

15. Morrison VA. Echinocandin antifungals: review and update. Expert Rev Ant Infect Ther. 2006:4:325-42.

16. Limper AH, Knox KS, Sarosi GA, Ampel NM, Bennett JE, Catanzaro A, Davies SF, Dismukes WE, Hage CA, Marr KA, Mody CH, Perfect JR, Stevens DA, on behalf of the American Thoracic Society Fungal Working Group. An Official American Thoracic Society Statement: Treatment of Fungal Infections in Adult Pulmonary and Critical Care Patients. Am J Respir Crit Care Med. 2011; 183:96-128.

Submit your next manuscript to BioMed Central and we will help you at every step:

- We accept pre-submission inquiries

- Our selector tool helps you to find the most relevant journal

- We provide round the clock customer support

- Convenient online submission

- Thorough peer review

- Inclusion in PubMed and all major indexing services

- Maximum visibility for your research

Submit your manuscript at www.biomedcentral.com/submit 